\title{
Cirugía de revascularización miocárdica versus angioplastía coronaria con stent en enfermedad de tres vasos y/o tronco común izquierdo en diabéticos: meta-análisis de estudios aleatorios
}

\author{
ANDRÉS JADUE T. ${ }^{1}$, ROBERTO GONZÁLEZ L. ${ }^{2}$, \\ MANUEL J. IRARRÁZAVAL LL. ${ }^{1}$
}

'División de Enfermedades

Cardiovasculares, Facultad de Medicina, Pontificia Universidad Católica de Chile, Santiago, Chile.

${ }^{2}$ Equipo de Cirugía Cardiotorácica, Hospital

"Dr. Guillermo Grant Benavente", Facultad de Medicina, Universidad de Concepción, Concepción, Chile.

No existen conflictos de interes ni apoyo financiero

Recibido el 27 de octubre de 2011, aceptado el 29 de enero de 2012.

Correspondencia a: Andrés Jadue Tobar

División de Enfermedades Cardiovasculares, Facultad de Medicina, Pontificia Universidad Católica de Chile, Santiago, Chile. Marcoleta $367,8^{\circ}$ piso Fono: 056-02-3453231 Fax: 056-02-6390108 E-mail: ajjadue@uc.cl

\section{Meta-analysis of coronary artery bypass surgery compared to percutaneous transluminal angioplasty with stent in diabetic patients}

Background: Diabetic patients are a group of primary interest in the study of myocardial revascularization. Aim: To compare coronary artery bypass grafting surgery $(C A B G)$ and percutaneous angioplasty with stents (PCI-S) in diabetic patients with coronary three-vessel or left main coronary artery disease. Material and $\mathbf{M e -}$ thods: Meta-analysis of MEDLINE randomized controlled studies comparing CABG and PCI-S in diabetic patients. The primary outcome measure was major adverse cardiovascular events (MACCE), death, myocardial infarction, cerebrovascular accident (CVA) and coronary re-intervention. Secondary outcomes were the individual components of MACCE. Results: Three studies comparing CABG and PCI-S met the inclusion criteria. One thousand sixty two patients were studied: 565 in the $C A B G$ group and 597 in the PCI-S group. At one year follow up MACCE occurred in 24.9 and $12.7 \%$ of patients in PCI-S and CABG groups, respectively (Odds ratio (OR) 2.27; 95\% confidence intervals (CI) 1.66-3.09). There were no differences in death or myocardial infarction. Strokes were less common in the PCI-S group (OR 0.25, 95\% CI 0.09-0.68) and coronary re-intervention was required with higher frequency in the PCI-S group (OR 5.32, 95\% CI 3.27-8.67). Conclusions: In diabetic patients with three-vessel coronary disease or left main coronary artery, revascularization with CABG had significantly less MACCE at one year than those treated with PCI-S. Stroke frequency was higher in $C A B G$, coronary re-intervention was higher in PCI-S. These results must be interpreted cautiously.

(Rev Med Chile 2012; 140: 640-648).

Key words: Angioplasty; Coronary artery bypass; Coronary artery disease.
L

a diabetes mellitus (DM) es una enfermedad con creciente prevalencia en Chile y en el mundo, además de un importante factor de riesgo cardiovascular ${ }^{1,2}$. Los pacientes con DM presentan una ateroesclerosis difusa y rápidamente progresiva, esto los expone a un riesgo 2 a 4 veces mayor de enfermedad coronaria y es responsable de aproximadamente el 75\% de las muertes relacionadas a la $\mathrm{DM}^{3}$.

$\mathrm{Al}$ menos un cuarto de los pacientes referidos a revascularización miocárdica presentan DM y los resultados clínicos del tratamiento médico, intervencionista o quirúrgico son inferiores que en pacientes no diabéticos ${ }^{4}$. 
El tratamiento de elección en la enfermedad coronaria de tres vasos y/o tronco común izquierdo es la cirugía de revascularización miocárdica $(\mathrm{CABG})$. Las guías clínicas vigentes clasifican esta recomendación como nivel de evidencia "A", es decir, respaldada por más de un estudio controlado aleatorio $(\mathrm{RCT})^{5,6}$.

Estudios clínicos han demostrado mejor supervivencia en pacientes diabéticos tratados con $C A B G$ versus angioplastia percutánea (PCI). El subgrupo de diabéticos ha adquirido especial relevancia desde la publicación de los resultados del $\mathrm{BARI}^{7}$ y hoy en día constituye un grupo de principal interés en el estudio de la revascularización miocárdica.

La PCI ha mejorado los resultados con la introducción de stents (PCI-S), metálicos (BMS) o medicados (DES), inhibidores de la glicoproteína $\mathrm{IIb} / \mathrm{III}$ y nuevos antiagregantes plaquetarios ${ }^{8}$.

La selección de la estrategia de revascularización óptima en pacientes con enfermedad coronaria y DM es crucial para mejorar los resultados clínicos en este grupo de pacientes de riesgo. A pesar de los múltiples estudios controlados que se han realizado comparando CABG versus PCI$S$, no encontramos meta-análisis en la literatura que comparen los resultados de estas estrategias de revascularización en la subpoblación diabética.

Nuestro objetivo es realizar un meta-análisis de estudios controlados que comparen los resultados de CABG versus PCI-S en pacientes diabéticos con enfermedad coronaria de tres vasos y/o tronco común izquierdo.

\section{Materiales y Método}

Se siguieron las recomendaciones vigentes del grupo QUORUM 9 .

Se realizó una búsqueda en MEDLINE de estudios publicados desde enero de 2000 hasta julio de 2010 utilizando los términos Mesh (Medical Subterm ) "Diabetes Mellitus", "Coronary Artery Bypass", "Angioplasty, Transluminal, Percutaneous Coronary". Se seleccionaron artículos adicionales en base a las referencias de los artículos encontrados, revisiones o meta-análisis previos y consultas a expertos.

Se seleccionaron estudios en base a los siguientes criterios de inclusión:

1) Publicados en revistas indexadas con disponibilidad de texto completo en inglés o español.
2) RCT que comparasen CABG versus PCI-S (BMS o DES) en enfermedad coronaria de tres vasos o enfermedad de tronco común izquierdo.

3) Incluyeran en el estudio sólo pacientes diabéticos o publicaran de manera detallada los resultados del subgrupo de pacientes diabéticos.

4) El seguimiento de los pacientes fuera al menos de 12 meses.

El resultado primario estudiado fueron los eventos adversos cardiovasculares mayores (MACCE), definidos como el evento conjunto de muerte, infarto miocárdico, accidente cerebrovascular (ACV) y necesidad de reintervención coronaria. Los resultados secundarios estudiados fueron los componentes individuales de los MACCE, es decir, muerte, infarto miocárdico, ACV y reintervención por separado.

La calidad metodológica de los estudios incluidos se categorizó mediante la puntuación de Jadad y cols ${ }^{10}$.

Los datos clínicos predefinidos fueron obtenidos de los estudios seleccionados, incluyendo las características clínicas basales de los pacientes y los resultados. Dos revisores (AJT y RGL) independientemente extrajeron los datos del texto, tablas y figuras de los artículos. En caso de no haber consenso entre los autores se obtuvo consenso en base al tercer autor (MJI).

El meta-análisis se realizó con el programa Review Manager (RevMan) [Computer program]. Version 5.0. Copenhagen: The Nordic Cochrane Centre, The Cochrane Collaboration, 2008. Se calculó el Odds Ratio (OR) y su intervalo de confianza a 95\% (IC95\%) para cada uno de los resultados predeterminados que compararan CABG versus PCI-S. Se realizaron "Forest Plots" para la presentación gráfica de los resultados. Todos los valores de $p$ fueron de 2-colas, se consideró significativo $<0,05$. La heterogeneidad entre los estudios se estudió mediante la prueba $\mathrm{I}^{2}$. Se consideró el grado de heterogeneidad como bajo $(<25 \%)$, moderado $(25-50 \%)$ y alto $(>50 \%)$.

\section{Resultados}

Con los criterios de búsqueda descritos se encontraron 157 artículos. De estos, 28 artículos fueron RCT, por lo que se excluyeron 129 estudios no aleatorios.

Se excluyeron 11 artículos aleatorios por com- 
parar intervenciones distintas a CABG versus PCI, 2 por comparar CABG versus $P C I$ en escenario de urgencia, 8 por comparar CABG versus PCI sin stent, 2 por no detallar los resultados de CABG versus PCI-S en la subpoblación diabética, 1 por comparar PCI-S (DES) contra una serie histórica randomizada entre PCI-S y CABG; y 1 estudio que cumple los criterios de inclusión, pero que aún no ha sido completado. Los detalles de la inclusión y exclusión de los artículos encontrados se resumen en la Figura 1.

Tres estudios cumplieron los criterios de inclusión al meta-análisis (ARTS, SYNTAX, CARDia $)^{11-13}$. Los tres estudios fueron catalogados de "alta calidad" según la puntuación de Jadad.

Un total de 1.162 pacientes fueron analizados; 565 en el grupo CABG y 597 en el grupo PCI-S. Sólo uno de estos estudios incluyó exclusivamente pacientes diabéticos ${ }^{13}$ y el tratamiento con PCI fue con BMS (31\%) o DES (69\%). Los otros dos artículos, fueron estudios que incluyeron una población más amplia, pero que detallaron sus resultados en los pacientes diabéticos ${ }^{11,12}$. De estos últimos en uno se utilizó BMS ${ }^{11}$ y en otro DES ${ }^{12}$.

Los pacientes tratados con angioplastía presentaron un riesgo significativamente mayor de MACCE un año luego de su intervención (OR 2,27; IC95\% 1,66-3,09; p < 0,001). El valor de $\mathrm{I}^{2}$ no demostró heterogeneidad estadística $\left(\mathrm{I}^{2}=0 \%\right)$ (Figura 2).

No hubo diferencias significativas en la mortalidad a un año post procedimiento (OR 1,26; IC95\% 0,79-2,33; $\mathrm{p}=0,26)$. El valor de $\mathrm{I}^{2}$ no demostró heterogeneidad estadística $\left(\mathrm{I}^{2}=0 \%\right)$ (Figura 3).

No hubo diferencias significativas en la presencia de infarto a un año post procedimiento (OR $1,54$; IC95\% 0,93-2,56; $\mathrm{p}=0,09)$. El valor de $\mathrm{I}^{2}$ no demostró heterogeneidad estadística $\left(\mathrm{I}^{2}=0 \%\right)$ (Figura 4).

Los pacientes revascularizados con PCI-S presentaron un riesgo significativamente menor de accidentes cerebrovasculares a un año post procedimiento (OR 0,25; IC95\% 0,09-0,68; p = 0,007). El valor de $\mathrm{I}^{2}$ no demostró heterogeneidad estadística $\left(\mathrm{I}^{2}=0 \%\right)$ (Figura 5$)$.

Los pacientes tratados con angioplastía presentaron un riesgo significativamente mayor de reintervenciones coronarias a un año post procedimiento (OR: 5,32; IC95\% 3,27 8,67; p < 0,001). El valor de $\mathrm{I}^{2}$ no demostró heterogeneidad estadística $\left(\mathrm{I}^{2}=0 \%\right)$ (Figura 6).

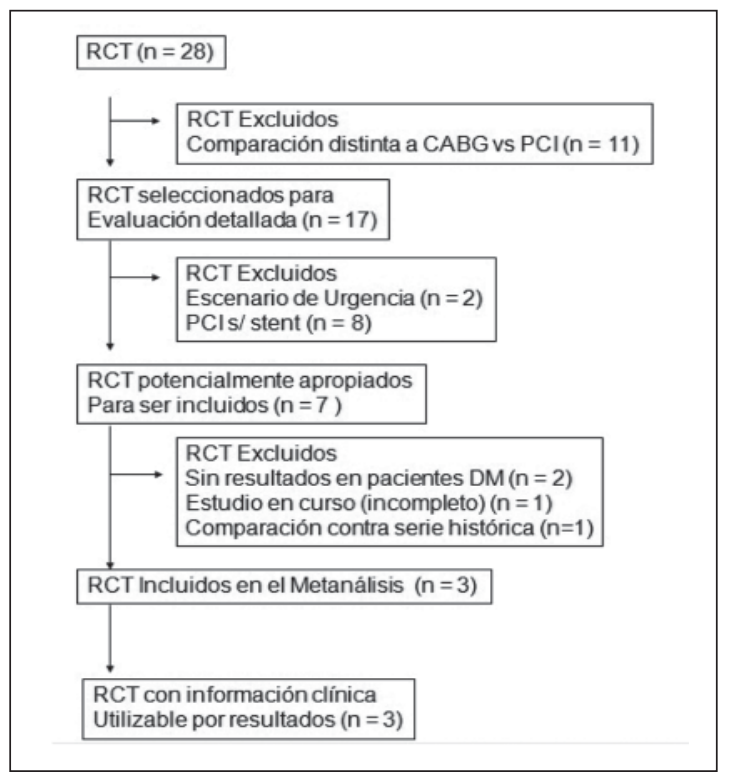

Figura 1. Diagrama de flujo de los estudios Incluidos en el Meta-análisis. $(\mathrm{RCT}=$ Estudio Clínico Aleatorio; $\mathrm{CABG}=$ Cirugía de Revascularización Miocárdica; $\mathrm{PCl}=$ Angioplastía Coronaria Percutánea; $\mathrm{DM}=$ Diabetes Mellitus).

\begin{tabular}{|c|c|c|c|c|c|c|c|c|}
\hline \multirow[b]{2}{*}{ Study or Subgroup } & \multicolumn{2}{|c|}{ PCI } & \multicolumn{2}{|c|}{ CABG } & \multirow[b]{2}{*}{ Weight } & \multirow{2}{*}{$\begin{array}{l}\text { Odds Ratio } \\
\text { M-H, Fixed, 95\% Cl }\end{array}$} & \multirow{2}{*}{\multicolumn{2}{|c|}{$\begin{array}{c}\text { Odds Ratio } \\
\text { M-H, Fixed, 95\% Cl }\end{array}$}} \\
\hline & Events & Total & Events & Total & & & & \\
\hline ARTS & 41 & 112 & 15 & 96 & $18.6 \%$ & $3.12[1.59,6.10]$ & & $\rightarrow-$ \\
\hline CARDia & 49 & 254 & 28 & 248 & $41.4 \%$ & $1.88[1.14,3.10]$ & & $\rightarrow-$ \\
\hline SYNTAX & 59 & 231 & 29 & 221 & $40.0 \%$ & $2.27[1.39,3.71]$ & & $\rightarrow-$ \\
\hline Total $(95 \% \mathrm{Cl})$ & & 597 & & 565 & $100.0 \%$ & $2.27[1.66,3.09]$ & & $\bullet$ \\
\hline Total events & 149 & & 72 & & & & & \\
\hline $\begin{array}{l}\text { Heterogeneity: } \mathrm{Chi}^{2} \\
\text { Test for overall effect }\end{array}$ & $\begin{array}{l}1.41, d f= \\
Z=5.17\end{array}$ & $\begin{array}{l}2(P= \\
(P<0.0\end{array}$ & $\begin{array}{l}0.50) ; 1^{2}= \\
00001)\end{array}$ & & & & $\begin{array}{cc}0.01 & 0.1 \\
& \text { Favours } \mathrm{PCl}\end{array}$ & $\begin{array}{c}10 \quad 100 \\
\text { Favours CABG }\end{array}$ \\
\hline
\end{tabular}

Figura 2. Resultado Primario: MACCE a 12 meses. 


\begin{tabular}{|c|c|c|c|c|c|c|c|}
\hline \multirow[b]{2}{*}{ Study or Subgroup } & \multicolumn{2}{|l|}{$\mathrm{PCl}$} & \multicolumn{2}{|c|}{ CABG } & \multirow[b]{2}{*}{ Weight } & \multirow{2}{*}{$\begin{array}{l}\text { Odds Ratio } \\
\text { M-H, Fixed, } 95 \% \mathrm{Cl}\end{array}$} & \multirow{2}{*}{$\begin{array}{c}\text { Odds Ratio } \\
\text { M-H, Fixed, } 95 \% \mathrm{Cl}\end{array}$} \\
\hline & Events & Total & Events & Total & & & \\
\hline ARTS & 7 & 112 & 3 & 96 & $13.1 \%$ & $2.07[0.52,8.22]$ & $\longrightarrow$ \\
\hline CARDia & 8 & 254 & 8 & 248 & $34.0 \%$ & $0.98[0.36,2.64]$ & $\longrightarrow$ \\
\hline SYNTAX & 19 & 231 & 13 & 221 & $52.9 \%$ & $1.43[0.69,2.98]$ & 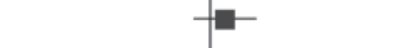 \\
\hline Total $(95 \% \mathrm{Cl})$ & & 597 & & 565 & $100.0 \%$ & $1.36[0.79,2.33]$ & \\
\hline Total events & 34 & & 24 & & & & \\
\hline \multicolumn{5}{|c|}{$\begin{array}{l}\text { Heterogeneity: } \mathrm{Ch}^{2}=0.80, \mathrm{df}=2(\mathrm{P}=0.67) ; \mathrm{I}^{2}=0 \% \\
\text { Test for overall effect: } Z=1.12(P=0.26)\end{array}$} & & & $\begin{array}{cccc}0.01 & 0.1 & 10 & 100 \\
& \text { Favours } \mathrm{PCl} & \text { Favours CABG }\end{array}$ \\
\hline
\end{tabular}

Figura 3. Resultado Secundario Mortalidad a 12 meses.

\begin{tabular}{|c|c|c|c|c|c|c|c|}
\hline Study or Subgroup & \multicolumn{2}{|c|}{ PCl } & \multicolumn{2}{|c|}{ CABG } & Weight & $\begin{array}{l}\text { Odds Ratio } \\
\text { M-H, Fixed, } 95 \% \mathrm{Cl}\end{array}$ & $\begin{array}{c}\text { Odds Ratio } \\
\text { M-H, Fixed, } 95 \% \mathrm{Cl}\end{array}$ \\
\hline ARTS & 7 & 112 & 3 & 96 & $12.3 \%$ & $2.07[0.52,8.22]$ & \\
\hline CARDia & 25 & 254 & 14 & 248 & $51.8 \%$ & $1.82[0.93,3.60]$ & \\
\hline SYNTAX & 9 & 231 & 9 & 221 & $35.9 \%$ & $0.95[0.37,2.45]$ & \\
\hline Total $(95 \% \mathrm{Cl})$ & & 597 & & 565 & $100.0 \%$ & $1.54[0.93,2.56]$ & \\
\hline Total events & 41 & & 26 & & & & \\
\hline $\begin{array}{l}\text { Heterogeneity: } \mathrm{Chi}^{2}= \\
\text { Test for overall effect }\end{array}$ & $\begin{array}{l}1.40, d f= \\
Z=1.68\end{array}$ & $\begin{array}{l}2(P= \\
(P=0 .\end{array}$ & $\begin{array}{l}0.50) ; 1^{2}= \\
9 \text { ) }\end{array}$ & $=0 \%$ & & & 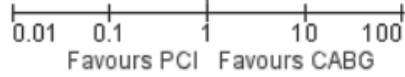 \\
\hline
\end{tabular}

Figura 4. Resultado Secundario Infarto Miocárdico a 12 meses.

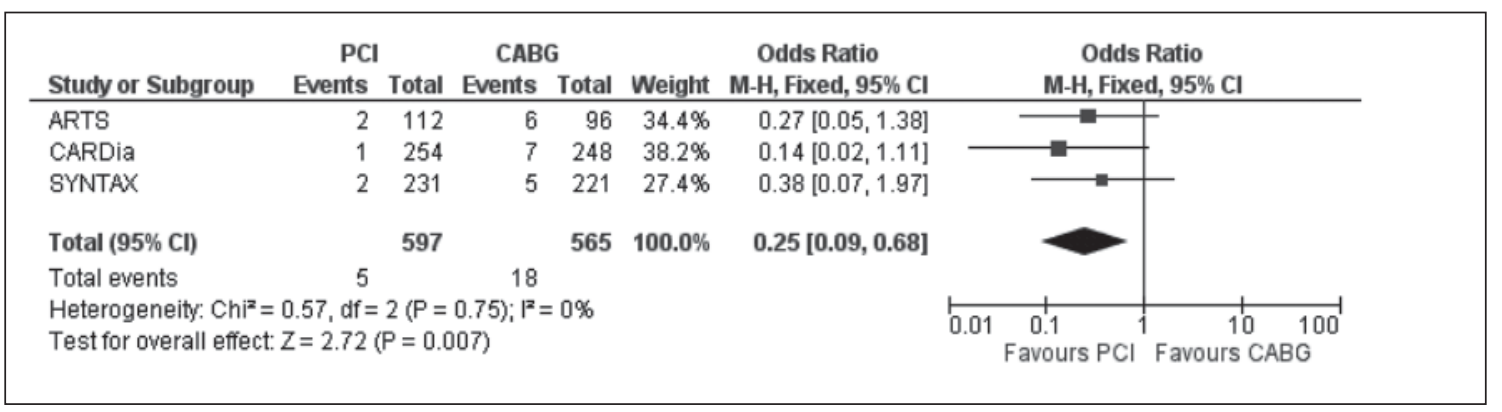

Figura 5. Resultado Secundario Accidente cerebrovascular a 12 meses.

\begin{tabular}{|c|c|c|c|c|c|c|c|c|}
\hline Study or Subgroup & \multicolumn{2}{|l|}{ PCI } & \multicolumn{2}{|c|}{ CABG } & Weight & $\begin{array}{l}\text { Odds Ratio } \\
\text { M-H, Fixed, } 95 \% \mathrm{Cl}\end{array}$ & \multicolumn{2}{|c|}{$\begin{array}{c}\text { Odds Ratio } \\
\text { M-H, Fixed, 95\% Cl }\end{array}$} \\
\hline ARTS & 25 & 112 & 3 & 96 & $14.2 \%$ & $8.91[2.60,30.56]$ & & $\longrightarrow$ \\
\hline CARDia & 30 & 254 & 5 & 248 & $25.3 \%$ & $6.51[2.48,17.07]$ & & $\longrightarrow$ \\
\hline SYNTAX & 46 & 231 & 13 & 221 & $60.4 \%$ & $3.98[2.08,7.60]$ & & \\
\hline Total $(95 \% \mathrm{Cl})$ & & 597 & & 565 & $100.0 \%$ & $5.32[3.27,8.67]$ & & \\
\hline Total events & 101 & & 21 & & & & & \\
\hline \multicolumn{5}{|c|}{$\begin{array}{l}\text { Heterogeneity. } \mathrm{Chi}^{2}=1.62, \mathrm{df}=2(\mathrm{P}=0.45) ;\left.\right|^{2}=0 \% \\
\text { Test for overall effect: } Z=6.71(\mathrm{P}<0.00001)\end{array}$} & & & $\begin{array}{lcl}0.01 & 0.1 \\
\text { Favours } \mathrm{PCl}\end{array}$ & $\begin{array}{c}10 \\
\text { Favours CABG }\end{array}$ \\
\hline
\end{tabular}

Figura 6. Resultado Secundario Reintervención a 12 meses. 


\section{Discusión}

La DM es una enfermedad frecuente y con significativas repercusiones cardiovasculares ${ }^{14}$. Se estima que su prevalencia se duplicará para el año 2030, principalmente en relación al envejecimiento de la población, la obesidad y el sedentarismo ${ }^{15}$. Independientemente de otros factores de riesgo, la DM es un importante factor de riesgo coronario y cerca de $75 \%$ de los pacientes con DM fallece por causas cardiovasculares ${ }^{3}$. Los efectos fisiopatológicos de la hiperglicemia/hiperinsulinemia, disfunción endotelial, aumento de la actividad plaquetaria, anormalidades en los lípidos y de las consecuencias de la inflamación vascular produce una enfermedad coronaria más difusa, más temprana y con peores resultados luego del tratamiento médico, intervencionista o quirúrgico ${ }^{16}$. Los pacientes diabéticos que son revascularizados mediante cirugía o angioplastía tienen una mayor mortalidad a largo plazo al compararlos con los pacientes no diabéticos ${ }^{17}$.

El estudio BARI (Bypass Angioplasty Revascularization Investigation) comparó la supervivencia a 5 años de pacientes revascularizados mediante CABG versus PCI con balón. Los resultados demostraron una supervivencia a 7 años de $84,4 \%$ para CABG versus $80,9 \%$ para el grupo PCI $(\mathrm{p}=0,043)$, principalmente dependiente de 353 pacientes diabéticos en quienes la supervivencia con CABG $(76,4 \%)$ fue significativamente mejor que con PCI $(55,7 \%)(\mathrm{p}<0,001)^{7}$. En el estudio SYNTAX (SYnergy Beetwen PCI with TAXus and Cardiac Surgery) la presencia de DM se asoció a un aumento de la mortalidad independientemente de la estrategia de revascularización empleada. Los resultados a un año no demostraron una diferencia significativa en la mortalidad cuando se comparó PCI-S versus CABG, salvo para lesiones complejas $(\text { Puntaje SYNTAX } \geq 33)^{18}$.

Los resultados de nuestro meta-análisis no demostraron una diferencia significativa en cuanto a la mortalidad ni infarto miocárdico, aunque se observa una tendencia que no alcanza significación estadística. Estos resultados deben analizarse con cautela por las siguientes razones:

1. Los estudios incluidos fueron diseñados para encontrar diferencias significativas en MACCE (que corresponde a una variable compuesta), por lo que la ausencia de diferencias significativas entre los eventos por separado (muerte, infarto miocárdico) no significa que no existan en la realidad. Por lo anteriormente señalado el resultado primario estudiado de este meta-análisis es acorde al resultado primario estudiado en cada uno de los estudios controlados incluidos, es decir, MACCE, donde si se demostraron diferencias estadísticamente significativas. De hecho, siguiendo el diseño del SYNTAX ${ }^{19}$ (nivel de significación de 5\% una cola, poder estadístico de 90\%), para encontrar $2 \%$ de diferencia en la mortalidad a un año se necesitaría un estudio de al menos 6.000 pacientes.

2. Evaluar resultados con un año de seguimiento podría resultar insuficiente desde el punto de vista clínico y estadístico. Ambas estrategias de revascularización se han convertido en técnicas seguras, reduciendo el número de eventos peri-procedimiento, por lo que registrar eventos clínicos significativos requiere de un seguimiento más prolongado. Por otro lado, realizar un seguimiento a un año podría ir en desmedro del principal beneficio de la revascularización quirúrgica, cuyos resultados alejados son un bien probado ${ }^{20} . \mathrm{Al}$ analizar los resultados del estudio SYNTAX a un año de seguimiento, la incidencia de infartos post procedimientos mostraron diferencias no significativas $(3,3 \%$ para CABG, $4,8 \%$ para PCI; $\mathrm{p}=0,11)$. Sin embargo, al completar 3 años de seguimiento los pacientes tratados con CABG tuvieron significativamente menos infartos miocárdicos que los pacientes tratados con PCI $(3,6 \% \text { vs } 7,3 \% ; \mathrm{p}=0,002)^{21}$.

La presencia de MACCE en cada uno de los estudios reclutados fue mayor para el grupo de los PCI-S, y finalmente también fue mayor para el riesgo final (Figura 2), principalmente a causa de una mayor necesidad de reintervención en el grupo de PCI-S.

La necesidad de reintervención es frecuentemente menospreciada en la literatura y en la práctica clínica, llegando a denominar "hard MACCE" a la variable conjunta de muerte, infarto o $\mathrm{ACV}^{22}$. Sin embargo, la presencia de reintervención, es un evento clínico significativo, que implica una falla en el tratamiento mediante persistencia de angor o incluso algún evento agudo que amerita una reintervención con un deterioro significativo y documentado en la calidad de vida ${ }^{23}$. 
Las guías clínicas vigentes recomiendan que el tratamiento de elección para la enfermedad coronaria de tres vasos y/o tronco común izquierdo es la cirugía de revascularización miocárdica ${ }^{5,6}$. Esta recomendación tiene un nivel de evidencia tipo "A" dado que está respaldado en múltiples RCT. Frecuentemente en la preparación de guías clínicas se clasifica de manera jerárquica la evidencia clínica disponible, donde los RCT y sus meta-análisis son la cúspide de la pirámide ${ }^{24}$.

No hay que dejar pasar las limitantes metodológicas intrínsecas que tienen los RCT, que pueden mermar su validez externa, es decir, su aplicabilidad a la población general. Dada la extrema rigurosidad de inclusión a los estudios controlados aleatorios, sólo una pequeña proporción de los pacientes son incluidos en el estudio y finalmente estos pacientes tienen un espectro de enfermedad menos grave que no es representativa de la población a la cual se intenta extrapolar el estudio ${ }^{25}$. De hecho sólo entre 2 a $5 \%$ de los pacientes tamizados fueron incluidos en los primeros estudios controlados que compararon PCI vs $\mathrm{CABG}^{26} \mathrm{y}$, si se toman en cuenta los números publicados en el "run in phase", el estudio SYNTAX sólo incluye 4\% de la población potencialmente incluible ${ }^{27}$. Finalmente, de los 3.075 pacientes que eventualmente se ingresarían sólo 1.800 se aleatorizaron, ya que podían ser revascularizados con ambos métodos. De estos 1.275 pacientes no revascularizables por ambos métodos, 1.077 se incluyeron en un registro CABG dado su enfermedad coronaria compleja y 198 al registro PCI principalmente por sus comorbilidades que hacían demasiado riesgosa la cirugía de revascularización. Al año de seguimiento 8,8\% de los pacientes del registro CABG y 20,4\% del registro PCI habían presentado MACCE ${ }^{28}$.

Estas falencias intrínsecas en la metodología de los RCT han hecho considerar publicaciones médicas basadas en grandes series retrospectivas complementando información clínica de los estudios controlados para tener resultados más representativos del "mundo real" 25 .

En estas grandes series "observacionales", por ejemplo la de Edward L. Hannan en Nueva York, que incluye más de 59.000 pacientes con enfermedad coronaria de dos o más vasos y que compara PCI con BMS versus CABG, el grupo tratado con CABG presentó una supervivencia alejada significativamente mayor que el grupo tratado con PCI. En este registro se incluyen 1.310 pacientes diabéticos con enfermedad de tres vasos tratados con PCI y 8.939 tratados con CABG, en que se demostró un aumento de la supervivencia en los pacientes diabéticos tratados con cirugía ${ }^{29}$.

El mismo autor repite la experiencia comparando CABG versus PCI con DES en más de 17.000 pacientes con similares resultados, aunque la tendencia del aumento de la supervivencia global o libre de infarto en los pacientes diabéticos no es significativa estadísticamente (HR ajustado 0,84 IC 95\% 0,69-1,01; $\mathrm{p}=0,07)$, sin embargo, hay que considerar que el seguimiento es sólo de 18 meses $^{30}$.

Generalmente los resultados obtenidos de las series retrospectivas son desestimados cuando se obtienen resultados contradictorios a los $\mathrm{RCT}^{24}$. Una de las razones por las que estos resultados pueden diferir de lo observado en los RCT es por los sesgos de selección de tratamiento, que son minimizados con el proceso de aleatorización. Otra razón es por la falta de validez externa de los RCT, que por sus exigentes criterios de inclusión, los pacientes seleccionados no sean representativos de los pacientes estudiados, si no francamente menos enfermos. Finalmente, el diseño metodológico de un RCT tiene por objetivo determinar diferencias (o similitudes) para un resultado con cierto poder estadístico, y cuando este resultado es una variable compuesta (como MACCE) frecuentemente carecen de un poder estadístico suficiente para encontrar diferencias en variables menos frecuentes. Esto puede llevar a conclusiones erradas, especialmente falsos negativos, donde ampliando el número de pacientes estudiados o el período de seguimiento dejarían descubiertas diferencias significativas ${ }^{25}$.

El presente meta-análisis de RCT es calificado como evidencia tipo "A", es decir, la más alta jerárquicamente en la evidencia médica, pero no está exenta de limitaciones. Sumada a las limitaciones ya mencionadas de incluir sólo RCT que podrían no representar a la población real estudiada ("mundo real") se suman algunas limitaciones propias de este meta-análisis.

El seguimiento a un año es breve, y podría ser insuficiente para detectar diferencias entre ambos procedimientos, pues el rendimiento de los puentes coronarios y de los stents se hace más divergente en el tiempo, a favor de la cirugía.

La subpoblación diabética es un grupo de pacientes de interés, pero la extracción de estos datos es un poco "forzada". De los 3 trabajos incluidos, 
sólo uno se limita a los pacientes diabéticos (CARDia) mientras que los restantes (SYNTAX, ARTS) incluyen una población más grande y detallan $a$ posteriori los resultados en la subpoblación diabética, por lo que resultados de estudios en curso como el FREEDOM (Future REvascularization Evaluation in patients with Diabetes mellitas: Optimal management of Multivessel disease) se hacen cada vez más necesarios ${ }^{31}$. Otros estudios que comparan CABG versus PCI-S (BMS o DES) no presentan los resultados en sus pacientes diabéticos y restringen cuantitativamente el meta-análisis ${ }^{32-34}$.

Finalmente, con el advenimiento de nuevas tecnologías, la PCI ha logrado disminuir complicaciones intrínsecas como la oclusión aguda con el uso de BMS y la hiperplasia intimal con los DES ${ }^{35}$. Los estudios de este meta-análisis incluye pacientes con BMS (ARTS), DES (SYNTAX) o ambos (CARDia), y por la presentación de los resultados en la literatura, no permite estratificar los pacientes con BMS o DES y analizarlos por separado.

La CABG constituye el tratamiento de elección en la enfermedad coronaria de tres vasos y/o tronco común izquierdo, y los pacientes diabéticos son un grupo especialmente beneficiado. A pesar de esto, la práctica clínica difiere de las recomendaciones en el día a día. Los resultados de la cirugía a largo plazo son seguros y conocidos. La PCI, aparece como una alternativa menos invasiva, pero en ninguna de sus variedades (balón, BMS, DES) ha demostrado igualar los resultados de la cirugía de revascularización miocárdica ${ }^{36}$.

En el momento de elegir la aproximación terapéutica para la enfermedad coronaria, el "equipo cardiaco" (cardiólogo clínico, cardiólogo intervencionista, cirujano cardiovascular) y el paciente deben estar concientes del rendimiento de cada uno de los tratamientos.

\section{Conclusión}

En los pacientes diabéticos con enfermedad coronaria de tres vasos y/o tronco común izquierdo, la revascularización con CABG presenta significativamente menos MACCE a un año que los tratados con PCI-S. Los ACV fueron mayor en el grupo CABG, la necesidad de reintervención fue mayor en el grupo tratado con PCI-S. La interpretación de estos resultados debe realizarse con cautela y su extrapolación a la población general carece de justificación metodológica.
Agradecimientos: Agradecemos a los Doctores Fernando Altermatt y Jorge Dagnino por la asesoría metodológica brindada en la preparación y revisión del meta-análisis.

\section{Referencias}

1. Mokdad AH, Ford ES, Bowman BA, Dietz WH, Vinicor F, Bales VS, et al. Prevalence of obesity, diabetes, and obesity-related health risk factors, 2001. JAMA 2003; 289: 76-9.

2. Yusuf S, Hawken S, Ounpuu S, Dans T, Avezum A, Lanas F, et al. Effect of potentially modifiable risk factors associated with myocardial infarction in 52 countries (the INTERHEART study): case-control study. Lancet 2004; 364: 937-52.

3. Lüscher TF, Creager MA, Beckman JA, Cosentino F. Diabetes and vascular disease: pathophysiology, clinical consequences, and medical therapy: Part II. Circulation 2003; 108: 1655-61.

4. Flaherty JD, Davidson CJ. Diabetes and coronary revascularization. JAMA 2005; 293: 1501-8.

5. Eagle KA, Guyton RA, Davidoff R, Edwards FH, Ewy GA, Gardner TJ, et al. ACC/AHA 2004 guideline update for coronary artery bypass graft surgery: a report of the American College of Cardiology/American Heart Association Task Force on Practice Guidelines (Committee to Update the 1999 Guidelines for Coronary Artery Bypass Graft Surgery). Circulation 2004; 110: e340-e437.

6. Task Force on Myocardial Revascularization of the European Society of Cardiology (ESC) and the European Association for Cardio-Thoracic Surgery (EACTS); European Association for Percutaneous Cardiovascular Interventions (EAPCI), Kolh P, Wijns W, Danchin N, Di Mario C, Falk V, Folliguet T, et al. Guidelines on myocardial revascularization. Eur J Cardiothorac Surg 2010; 38: S1-S52.

7. The BARI Investigators. Seven-year outcome in the Bypass Angioplasty Revascularization Investigation (BARI) by treatment and diabetic status. J Am Coll Cardiol 2000; 35: 1122-9.

8. Babapulle MN, Joseph L, Bélisle P, Brophy JM, Eisenberg MJ. A hierarchical Bayesian meta-analysis of randomised clinical trials of drug-eluting stents. Lancet 2004; 364: 583-91.

9. Moher D, Cook DJ, Eastwood S, Olkin I, Rennie D, Stroup DF. Improving the quality of reports of metaanalyses of randomised controlled trials: the QUOROM statement. Quality of Reporting of Meta-analyses. Lancet 1999; 354: 1896-900. 
10. Jadad AR, Moore RA, Carroll D, Jenkinson C, Reynolds DJ, Gavaghan DJ, et al. Assessing the quality of reports of randomized clinical trials: is blinding necessary? Control Clin Trials 1996; 17: 1-12.

11. Abizaid A, Costa MA, Centemero M, Abizaid AS, Legrand VM, Limet RV, et al. Clinical and economic impact of diabetes mellitus on percutaneous and surgical treatment of multivessel coronary disease patients: insights from the Arterial Revascularization Therapy Study (ARTS) trial. Circulation 2001; 104: 533-8.

12. Banning AP, Westaby S, Morice MC, Kappetein AP, Mohr FW, Berti S, et al Diabetic and nondiabetic patients with left main and/or 3-vessel coronary artery disease: comparison of outcomes with cardiac surgery and paclitaxeleluting stents. J Am Coll Cardiol 2010; 55: 1067-75.

13. Kapur A, Hall RJ, Malik IS, Qureshi AC, Butts J, de Belder M, et al. Randomized comparison of percutaneous coronary intervention with coronary artery bypass grafting in diabetic patients. 1-year results of the CARDia (Coronary Artery Revascularization in Diabetes) trial. J Am Coll Cardiol 2010; 55: 432-40.

14. Elsässer A, Möllmann H, Nef HM, Hamm CW. How to revascularize patients with diabetes mellitus: bypass or stents and drugs? Clin Res Cardiol 2006; 95: 195-203.

15. Boyle JP, Honeycutt AA, Narayan KM, Hoerger TJ, Geiss LS, Chen $\mathrm{H}$, et al. Projection of diabetes burden through 2050: impact of changing demography and disease prevalence in the U.S. Diabetes Care 2001; 24: 1936-40.

16. Creager MA, Lüscher TF, Cosentino F, Beckman JA. Diabetes and vascular disease: pathophysiology, clinical consequences, and medical therapy: Part I. Circulation 2003; 108: 1527-32.

17. Lee MS, Yang T, Dhoot J, Iqbal Z, Liao H. Meta-analysis of studies comparing coronary artery bypass grafting with drug-eluting stenting in patients with diabetes mellitus and multivessel coronary artery disease. Am J Cardiol 2010; 105: 1540-4.

18. Serruys PW, Morice MC, Kappetein AP, Colombo A, Holmes DR, Mack MJ, et al. SYNTAX Investigators. Percutaneous coronary intervention versus coronaryartery bypass grafting for severe coronary artery disease. N Engl J Med 2009 5; 360: 961-72.

19. Ong AT, Serruys PW, Mohr FW, Morice MC, Kappetein AP, Holmes DR, et al. The SYNergy between percutaneous coronary intervention with TAXus and cardiac surgery (SYNTAX) study: design, rationale, and run-in phase. Am Heart J 2006; 151: 1194-204.

20. Lytle BW, Blackstone EH, Loop FD, Houghtaling PL, Arnold JH, Akhrass R, et al. Two internal thoracic artery grafts are better than one. J Thorac Cardiovasc Surg 1999; 117: 855-72.
21. Kappetein AP, Feldman TE, Mack MJ, Morice MC, Holmes DR, Ståhle E, et al Comparison of coronary bypass surgery with drug-eluting stenting for the treatment of left main and/or three-vessel disease: 3 -year follow-up of the SYNTAX trial. Eur Heart J 2011; 32: 2125-34.

22. Hoffman SN, TenBrook JA, Wolf MP, Pauker SG, Salem DN, Wong JB. A meta-analysis of randomized controlled trials comparing coronary artery bypass graft with percutaneous transluminal coronary angioplasty: one- to eight-year outcomes. J Am Coll Cardiol 2003 16; 41: 1293-304.

23. Cohen DJ, Van Hout B, Serruys PW, Mohr FW, Macaya C, den Heijer P, et al. Quality of life after PCI with drugeluting stents or coronary-artery bypass surgery. N Engl J Med 2011; 364: 1016-26.

24. Brown ML, Gersh BJ, Holmes DR, Bailey KR, Sundt TM 3rd. From randomized trials to registry studies: translating data into clinical information. Nat Clin Pract Cardiovasc Med 2008; 5: 613-20.

25. Hannan EL. Randomized clinical trials and observational studies: guidelines for assessing respective strengths and limitations. JACC Cardiovasc Interv 2008; 1: 211-7.

26. Guyton RA. Coronary artery bypass is superior to drugeluting stents in multivessel coronary artery disease. Ann Thorac Surg 2006; 81: 1949-57.

27. Irarrázaval MJ. ¿Es relevante el estudio SYNTAX para la toma de decisiones en cardiología? Rev Med Chile 2009; 37: 1535-7.

28. Mohr F, Serryus P. The Synergy between Percutaneous Coronary Intervention with TAXUS and Cardiac Surgery: The SYNTAX Study. One Year Results of the PCI and CABG Registries (en línea) (fecha de acceso 27 de octubre de 2011). URL disponible en: http://www.clinicaltrialresults.org/Slides/SYNTAX\%20Registries\%20(3). ppt

29. Hannan EL, Racz MJ, Walford G, Jones RH, Ryan TJ, Bennett E, et al. Long-term outcomes of coronary-artery bypass grafting versus stent implantation. $\mathrm{N}$ Engl J Med 2005; 352: 2174-83.

30. Hannan EL, Wu C, Walford G, Culliford AT, Gold JP, Smith CR. Drug-eluting stents vs coronary-artery bypass grafting in multivessel coronary disease. N Engl J Med 2008; 358: 331-41.

31. Farkouh ME, Dangas G, Leon MB, Smith C, Nesto R, Buse JB, et al Design of the Future REvascularization Evaluation in patients with Diabetes mellitus: Optimal management of Multivessel disease (FREEDOM) Trial. Am Heart J 2008; 155: 215-23.

32. SoS Investigators. Coronary artery bypass surgery versus percutaneous coronary intervention with stent implan- 
Cirugía coronaria versus angioplastia con stent en diabéticos. Meta-análisis - A. Jadue et al

tation in patients with multivessel coronary artery disease (the Stent or Surgery trial): a randomised controlled trial. Lancet 2002; 360: 965-70.

33. Hueb W, Lopes NH, Gersh BJ, Soares P, Machado LA, Jatene FB, et al. Five-year follow-up of the Medicine, Angioplasty, or Surgery Study (MASS II): a randomized controlled clinical trial of 3 therapeutic strategies for multivessel coronary artery disease. Circulation 2007; 115: 1082-9.

34. Buszman PE, Kiesz SR, Bochenek A, Peszek-Przybyla E,
Szkrobka I, Debinski N, et al. Acute and late outcomes of unprotected left main stenting in comparison with surgical revascularization. J Am Coll Cardiol 2008; 51: $538-45$.

35. Garg S, Serruys PW. Coronary stents: current status. J Am Coll Cardiol 2010; 56: S1-42.

36. Reichenspurner H, Conradi L, Cremer J, Mohr FW. Best way to revascularize patients with main stem and three-vessel lesions. Patients should be operated. Clin Res Cardiol 2010; 99: 541-4. 